\title{
Identification of a Novel Arginine Vasopressin Receptor 2 Mutation (p.V183M) in a Chinese Family with Nephrogenic Diabetes Insipidus
}

\author{
Ji-Shi Liu ${ }^{a}$ Hao Huang ${ }^{b}$ Jie-Yuan Jin ${ }^{b}$ Ran Du ${ }^{b}$ Chen-Yu Wang ${ }^{b}$ \\ Liang-Liang Fan ${ }^{\mathrm{a}-\mathrm{c}}$ \\ a Department of Nephrology, The Third Xiangya Hospital of Central South University, Changsha, China; \\ ${ }^{b}$ Department of Cell Biology, The School of Life Sciences, Central South University, Changsha, China \\ 'Hunan Key Laboratory of Animal for Human Disease, School of Life Sciences, Central South University, \\ Changsha, China
}

\section{Keywords}

Arginine vasopressin receptor $2 \cdot$ Nephrogenic diabetes insipidus · Novel mutation

\begin{abstract}
Loss of function of arginine vasopressin receptor 2 (AVPR2) may affect the recognition and binding of arginine vasopressin (AVP) which, in turn, may prevent the activation of Gs/ adenylate cyclase and reduce the reabsorption of water by renal tubules and combined tubes. Finally, the organism may suffer from nephrogenic diabetes insipidus (NDI), a kind of kidney disorder featured by polyuria and polydipsia, due to a break of water homeostasis. In this study, we enrolled a Chinese family with polyuria and polydipsia. The proband presented abnormal fluid intake and excessive urine output. A water deprivation and AVP stimulation test further indicated that this patient had NDI. By sequencing known causative genes for diabetes insipidus, we identified a novel mutation in AVPR2 (c.547G >A; p.V183M) in the family. This mutation, located in a conserved site of AVPR2 and predicted to be disease-causing by informatics programs, was absent in our 200 controls and other public databases. Our study not only further confirms the clinical diagnosis, but also expands the spectrum of AVPR2 mutations and contributes to genetic diagnosis and counseling of patients with NDI.
\end{abstract}

(c) 2020 S. Karger AG, Basel

\section{KARGER}

$$
\text { ๑ } 2020 \text { S. Karger AG, Basel }
$$

Nephrogenic diabetes insipidus (NDI) is a rare kidney disorder related to water homeostasis. The main symptoms of NDI are polyuria and polydipsia, but some patients are also observed with vomiting, retching, unexplained fevers, lethargy, and irritability in early infancy [Bockenhauer and Bichet, 2015; Milano et al., 2017; Christ-Crain et al., 2019]. In comparison, acquired NDI is almost always less severe than the hereditary forms [Christ-Crain et al., 2019]. According to previous studies, most inherited NDI (approximately 90\%) had an Xlinked recessive pattern of inheritance, resulting from arginine vasopressin receptor 2 ( $A V P R 2$ ) mutations [Bichet and Bockenhauer, 2016]. In addition, autosomal recessive and autosomal dominant patterns of inheritance can both be present in NDI cases due to mutations in aquaporin 2 (AQP2) [Bichet and Bockenhauer, 2016; Milano et al., 2017]. Similarly, variants in arginine vasopressin $(A V P)$ and wolframin ER transmembrane glycoprotein (WFS1) can also lead to familial diabetes insipidus [Schernthaner-Reiter et al., 2017; Christ-Crain et al., 2019].

AVPR2 is located in $\mathrm{Xq} 28$ and encodes the vasopressin receptor 2 which belongs to the 7 -transmembrane-domain G protein-coupled receptor (GPCR) superfamily and cou-

J.-S.L. and H.H. contributed equally to this work. 


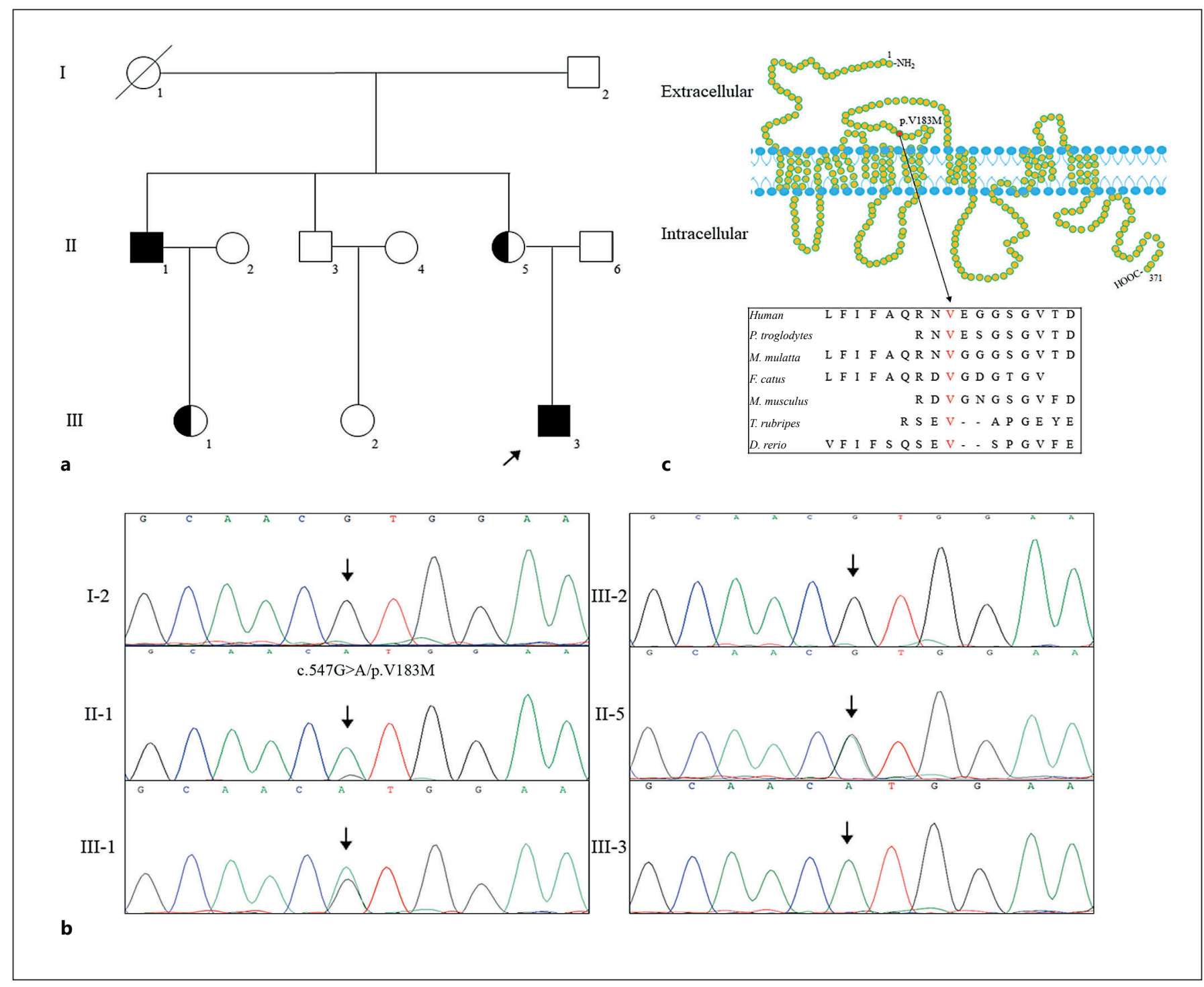

Fig. 1. a Pedigree of the family affected with X-linked dominant inherited NDI. Family members are identified by generations and numbers. Squares indicate male family members; circles, female members; black symbols, the affected members; open symbols, unaffected members; half black, the heterozygous carriers; arrow, proband. b Sequencing results of the AVPR2 mutation. Sequence chromatograms indicate a $\mathrm{G}$ to A transition of nucleotide 547. c Analysis of the mutation and protein domains of AVPR2 and alignment of multiple AVPR2 protein sequences across species. The affected amino acid V183 is located in the highly conserved amino acid region in different mammals (from Ensembl). Red letters show the V183 site. ples to Gs, thus stimulating adenylate cyclase [Milano et al., 2017; Kavanagh and Uy, 2019]. AVPR2 is expressed in the kidney tubule, especially in the distal convoluted tubule and collecting ducts [Grassmeyer et al., 2017]. The main function is to respond to the pituitary hormone AVP by stimulating mechanisms that concentrate the urine and maintain water balance in the organism [Milano et al.,
2017]. At present, more than 200 variants have been identified in NDI patients [Moeller et al., 2016].

In this study, we enrolled a Chinese NDI family with 2 patients and 7 healthy individuals. Genetic investigation and bioinformatics analyses suggested that a novel mutation in AVPR2 (c.547G>A; p.V183M) may be the genetic lesion of the family. 
Table 1. Results of the water deprivation and arginine vasopressin stimulation test

\begin{tabular}{rlllllll}
\hline Time, h & Weight, kg & $\begin{array}{l}\text { Heart rate, } \\
\text { beats/min }\end{array}$ & $\begin{array}{l}\text { Blood pressure, } \\
\mathrm{mmHg}\end{array}$ & $\begin{array}{l}\text { Urine volume, } \\
\mathrm{mL}\end{array}$ & $\begin{array}{l}\text { Urine specific } \\
\text { gravity }\end{array}$ & $\begin{array}{l}\text { Osmolality, } \\
\mathrm{mOsm} / \mathrm{kg} \mathrm{H} \mathrm{H}_{2} \mathrm{O}\end{array}$ \\
\hline 0 & 41 & 79 & $125 / 74$ & - & 1.0005 & 67 & $\begin{array}{l}\text { Serum osmolality, } \\
\mathrm{mOsm} / \mathrm{kg} \mathrm{H} \mathrm{H}_{2} \mathrm{O}\end{array}$ \\
2 & 41 & 81 & $120 / 70$ & 500 & 1.0005 & 73 & - \\
4 & 40.5 & 97 & $129 / 77$ & 450 & 1.0005 & 90 & - \\
6 & 40 & 122 & $117 / 83$ & 300 & 1.0005 & 99 & - \\
8 & 39.5 & 138 & $115 / 70$ & 300 & 1.0005 & 105 & 127 \\
10 & 39.5 & 84 & $110 / 68$ & 250 & 1.0006 & 154 & 355 \\
12 & 39.5 & 89 & $110 / 68$ & 100 & 1.0008 & 150 & 353 \\
13 & 40 & 88 & $120 / 74$ & 350 & 1.0007 & 109 & 320 \\
\hline
\end{tabular}

Desmopressin acetate $(5 \mathrm{U})$ was administered by subcutaneous injection $12 \mathrm{~h}$ after the basal investigation.

\section{Material and Methods}

\section{Subjects}

A 13-year-old boy was admitted to the Department of Nephrology, Third Xiangya Hospital, due to abnormal fluid intake and excessive urine output. In the past year, his water intake had increased from 3-4 L per day to 5-7 L per day, and urine output was more than 10 times per day. The patient reported that only liquid (water and milk) could appease thirst in his infancy. Family history investigation indicated that his uncle (II-1) also suffered from polyuria (5-6 times per night) and polydipsia (10-15 L per day) (Fig. 1a). The proband also accepted the water deprivation and arginine vasopressin stimulation test. Meanwhile, peripheral blood samples of 6 family members were enrolled (II-3 refused to participate).

\section{Mutation Detection and Cosegregation Analysis}

Genomic DNA was prepared from peripheral blood of the patients and all other participants using a DNeasy Blood \& Tissue Kit (Qiagen, Valencia, CA). The entire coding regions including the flanking intronic sequences of AVPR2 (Refseq: NM_000054), AQP2 (NM_000486), AVP (NM_000490), and WFS1 (NM_001145853) were amplified by PCR (primer sequences are available upon request). Sequences of the PCR products were determined using the ABI 3100 Genetic Analyzer (ABI, Foster City, CA) as previously described [Fan et al., 2019].

\section{Bioinformatics Analysis}

The multiple AVPR2 protein sequences across mammals were aligned using the program MUSCLE (version 3.6, http://www. ncbi.nlm.nih.gov). MutationTaster (www.mutationtaster.org) [Schwarz et al., 2014], SIFT (Sorting Intolerant From Tolerant, https://sift.bii.a-star.edu.sg/) [Ng and Henikoff, 2003], and PolyPhen-2 (polymorphism phenotyping, http://genetics.bwh.harvard.edu/pph2/) [Adzhubei et al., 2010] were used to predict the effect of the variant. The 1000 Genomes project (1000G, www.1000genomes.org), dbSNP132 (http://www.ncbi.nlm.nih. gov/projects/SNP/), ExAC (http://exac.broadinstitute.org/), and GenomAD databases (https://gnomad.broadinstitute.org/) were used to exclude the polymorphism sites.

\section{Results}

\section{Clinical Data}

Physical examination revealed that blood pressure (125/74 $\mathrm{mmHg}$ ), heart rate ( 79 beats/min), and skin turgor were normal in the proband. Laboratory data were as follows: urine specific gravity 1.0005 ; uric acid 466.3 $\mu \mathrm{mol} / \mathrm{L}$; HDL $0.97 \mathrm{mmol} / \mathrm{L}$; Na $146.3 \mathrm{mmol} / \mathrm{L} ; \mathrm{P} 1.69$ $\mathrm{mmol} / \mathrm{L}$; Mg $1.01 \mathrm{mmol} / \mathrm{L}$; glycated hemoglobin 5.3\%; normal thyroid function. The results of the water deprivation and arginine vasopressin stimulation test are shown in Table 1. During the water withdrawal period and after injection of desmopressin acetate, the urine specific gravity of the patient did not increase significantly $(<1.010)$. The highest urine osmotic pressure was 154 $\mathrm{mOsm} / \mathrm{kg}$, which was much lower than normal osmotic pressure (280-310 mOsm/kg). In addition, urine specific gravity and urine osmotic pressure of our patient did not improve after treatment by desmopressin acetate injection, which led to the hypothesis he might be a NDI patient.

\section{Genetic Analysis}

We then examined several genes known to cause diabetes insipidus. By sequencing analysis of $A V P R 2, A Q P 2$, $A V P$, and WFS1, a novel mutation in AVPR2 (c.547G $>$ A; p.V183M) was identified which cosegregated with the affected family members (Fig. 1b). This novel mutation, resulting in a substitution of valine by methionine, was absent in our 200 healthy controls [Xiang et al., 2014] as well as in the 1000 Genomes project, dbSNP132, ExAC, and GenomAD databases. Further bioinformatics analyses revealed that this mutation is located in a conserved site 
of the third extracellular domain (Fig. 1c) and is predicted to be deleterious by 3 different algorithm-based bioinformatics programs (MutationTaster, SIFT, and PolyPhen-2). Genetic analysis indicated that this novel mutation (c.547G>A; p.V183M) is the genetic lesion of the NDI family. According to ACMG guidelines, this mutation belongs to PS3+PM1+PM2 [Richards et al., 2015].

\section{Discussion and Conclusion}

According to previous studies, mutations in AVPR2 can lead to X-linked recessive NDI. Males with AVPR2 mutations are symptomatic, whereas heterozygous females are usually asymptomatic [Celebi Tayfur et al., 2018]. However, some heterozygous female carriers have been reported to have NDI; this may be due to random inactivation of the X chromosome [Zhang et al., 2019]. By binding AVPR2, AVP can activate Gs/adenylate cyclase and then regulate the water channel AQP2 in renal tubules and collecting ducts, and finally the concentration of urine [Caletti et al., 2014]. In this process, loss of AVPR2 function may induce a function disorder of the renal tubules and collecting ducts and thus break the water balance of the body [Erdelyi et al., 2015].

In our study, only 2 male family members (II- 1 and III-3) presented with NDI phenotypes, the proband's mother (II-5) and his cousin (III-1) were heterozygous carriers and were asymptomatic. The novel AVPR2 mutation (c.547G $>A ; p . V 183 \mathrm{M})$ is located in the third extracellular domain, which is responsible for AVP recognition and binding [Joshi et al., 2017]. This mutation may disrupt the structure of the extracellular domain and reduce the recognition and binding of AVP, and finally break the water homeostasis and lead to NDI. Our study is consistent with a previous study that mutations in the AVPR2 third extracellular domain can result in NDI [Joshi et al., 2018]. Similar mutations located in this domain have been reported in NDI patients, such as p.R181C and p.S187R [Pan et al., 1992; Boson et al., 2006].

At present, the treatment of NDI focuses on relieving symptoms, ensuring normal growth and development, preventing complications, and reducing adverse drug reactions [Di Iorgi et al., 2015]. Traditional treatment methods include drinking water, intravenous infusion, and avoiding water poisoning caused by heavy drinking. For underage patients, low-salt diet and reasonable protein diet are necessary to maintain growth and development [Bockenhauer and Bichet, 2015]. The traditional medicine for NDI are thiazide diuretics and a high concentra- tion of desmopressin [Konoshita et al., 2004; Sung and Lin, 2011; Schernthaner-Reiter et al., 2016]. In our study, the proband accepted the hydrochlorothiazide $(25 \mathrm{mg} /$ day) treatment, and the urine volume reduced to $2500 \mathrm{~mL}$ per day.

In summary, we report a novel mutation in $A V P R 2$ (c.547G>A; p.V183M) in a Chinese family with suspected NDI. Our analyses not only further confirmed the clinical diagnosis in the patients, but also expand the spectrum of AVPR2 mutations and contribute to genetic diagnosis and counseling of patients with NDI.

\section{Acknowledgements}

We thank all subjects for participating in this study. We thank Dr. Shuai Guo from University of Texas MD Anderson Cancer Center, USA, and Dr. Wan-Yun Tao from Case Western Reserve University, USA, for editing the language.

\section{Statement of Ethics}

The study was approved by the third Xiangya Hospital of the Central South University Ethics Committee and was performed according to the principles of the Declaration of Helsinki. Written informed consent was obtained from all participants.

\section{Disclosure Statement}

The authors have no conflicts of interest to declare.

\section{Funding Sources}

This study was supported by National Science and Technology Major Project of the Ministry of Science and Technology of China (2017ZX10103005-006), National Natural Science Foundation of China (81970403 and 81770002), and the Open Sharing Fund for the Large-Scale Instruments and Equipment of Central South University (CSUZC201940).

\section{Author Contributions}

J.-S.L. and H.H. carried out the sample collecting and genetic testing. J.-Y.J. and R.D. collected the clinical data. C.-Y.W. performed the bioinformatics analysis. J.-S.L. and L.-L. F. designed the project and wrote the manuscript, and L.-L.F. revised the manuscript. All authors read and approved the final manuscript. 


\section{References}

Adzhubei IA, Schmidt S, Peshkin L, Ramensky VE, Gerasimova A, et al: A method and server for predicting damaging missense mutations. Nat Methods 7:248-249 (2010).

Bichet DG, Bockenhauer D: Genetic forms of nephrogenic diabetes insipidus (NDI): vasopressin receptor defect (X-linked) and aquaporin defect (autosomal recessive and dominant). Best Pract Res Clin Endocrinol Metab 30:263-276 (2016)

Bockenhauer D, Bichet DG: Pathophysiology, diagnosis and management of nephrogenic diabetes insipidus. Nat Rev Nephrol 11:576-588 (2015).

Boson WL, Della Manna T, Damiani D, Miranda DM, Gadelha MR, et al: Novel vasopressin type 2 (AVPR2) gene mutations in Brazilian nephrogenic diabetes insipidus patients. Genet Test 10:157-162 (2006).

Caletti MG, Balestracci A, Di Pinto D: Pre- and post-treatment urinary tract findings in children with nephrogenic diabetes insipidus. Pediatr Nephrol 29:487-490 (2014).

-Celebi Tayfur A, Karaduman T, Ozcan Turkmen M, Sahin D, Caltik Yilmaz A, et al: A novel mutation in the AVPR2 gene causing congenital nephrogenic diabetes insipidus. J Clin Res Pediatr Endocrinol 10:350-356 (2018).

-Christ-Crain M, Bichet DG, Fenske WK, Goldman MB, Rittig S, et al: Diabetes insipidus. Nat Rev Dis Primers 5:54 (2019).

Di Iorgi N, Morana G, Napoli F, Allegri AE, Rossi A, Maghnie M: Management of diabetes insipidus and adipsia in the child. Best Pract Res Clin Endocrinol Metab 29:415-436 (2015).

Erdelyi LS, Mann WA, Morris-Rosendahl DJ, Gross U, Nagel M, et al: Mutation in the V2 vasopressin receptor gene, $A V P R 2$, causes nephrogenic syndrome of inappropriate diuresis. Kidney Int 88:1070-1078 (2015).
Fan LL, Ding DB, Huang H, Chen YQ, Jin JY, et al: A de novo mutation of SMYD1 (p.F272L) is responsible for hypertrophic cardiomyopathy in a Chinese patient. Clin Chem Lab Med 57:532-539 (2019).

Grassmeyer J, Mukherjee M, deRiso J, Hettinger $\mathrm{C}$, Bailey M, et al: Elf5 is a principal cell lineage specific transcription factor in the kidney that contributes to Aqp2 and Avpr2 gene expression. Dev Biol 424:77-89 (2017).

Joshi S, Brandstrom P, Gregersen N, Rittig S, Christensen JH: Novel de novo AVPR2 variant in a patient with congenital nephrogenic diabetes insipidus. Case Rep Nephrol Dial 7: 130-137 (2017).

Joshi S, Kvistgaard H, Kamperis K, Faerch M, Hagstrom $S$, et al: Novel and recurrent variants in AVPR2 in 19 families with X-linked congenital nephrogenic diabetes insipidus. Eur J Pediatr 177:1399-1405 (2018).

Kavanagh C, Uy NS: Nephrogenic diabetes insipidus. Pediatr Clin North Am 66:227-234 (2019).

Konoshita T, Kuroda M, Kawane T, Koni I, Miyamori I, et al: Treatment of congenital nephrogenic diabetes insipidus with hydrochlorothiazide and amiloride in an adult patient. Horm Res 61:63-67 (2004).

Dilano S, Carmosino M, Gerbino A, Svelto M, Procino G: Hereditary nephrogenic diabetes insipidus: pathophysiology and possible treatment. An update. Int J Mol Sci 18:E2385 (2017).

Moeller HB, Fuglsang CH, Fenton RA: Renal aquaporins and water balance disorders. Best Pract Res Clin Endocrinol Metab 30:277-288 (2016).
Ng PC, Henikoff S: SIFT: predicting amino acid changes that affect protein function. Nucleic Acids Res 31:3812-3814 (2003).

Pan Y, Metzenberg A, Das S, Jing B, Gitschier J: Mutations in the V2 vasopressin receptor gene are associated with X-linked nephrogenic diabetes insipidus. Nat Genet 2:103-106 (1992).

Richards S, Aziz N, Bale S, Bick D, Das S, et al: Standards and guidelines for the interpretation of sequence variants: a joint consensus recommendation of the American College of Medical Genetics and Genomics and the Association for Molecular Pathology. Genet Med 17:405-424 (2015).

Schernthaner-Reiter MH, Adams D, Trivellin G, Ramnitz MS, Raygada M, et al: A novel AVPR2 splice site mutation leads to partial Xlinked nephrogenic diabetes insipidus in two brothers. Eur J Pediatr 175:727-733 (2016).

Schernthaner-Reiter MH, Stratakis CA, Luger A: Genetics of diabetes insipidus. Endocrinol Metab Clin North Am 46:305-334 (2017).

Schwarz JM, Cooper DN, Schuelke M, Seelow D: MutationTaster2: mutation prediction for the deep-sequencing age. Nat Methods 11:361362 (2014).

Sung CC, Lin SH: Images in clinical medicine. Nonobstructive hydronephrosis with secondary polycythemia. N Engl J Med 365:e1 (2011).

Xiang R, Fan LL, Huang H, Cao BB, Li XP, et al: A novel mutation of GATA4 (K319E) is responsible for familial atrial septal defect and pulmonary valve stenosis. Gene 534:320-323 (2014).

Zhang M, Yu Q, Chen C, Han J, Cheng B, Tian D: A novel AVPR2 missense mutation in an Asian family with inherited nephrogenic diabetes insipidus: A case report. Medicine 98:e15348 (2019). 